\title{
STABILITY AND PERIODIC OSCILLATIONS IN THE MOON-RAND SYSTEMS
}

\author{
ADAM MAHDI, VALERY G. ROMANOVSKI, AND DOUGLAS S. SHAFER
}

\begin{abstract}
The Moon-Rand systems, developed to model control of flexible space structures, are systems of differential equations on $\mathbb{R}^{3}$ with polynomial or rational right hand sides that have an isolated singularity at the origin at which the linear part has one negative and one pair of purely imaginary eigenvalues for all choices of the parameters. We give a complete stability analysis of the flow restricted to a neighborhood of the origin in any center manifold of the Moon-Rand systems, solve the center problem on the center manifold, and find sharp bounds on the number of limit cycles that can be made to bifurcate from the singularity when it is a focus. We generalize the Moon-Rand systems in a natural way, solve the center problem in several cases, and provide sufficient conditions for existence of a center, which we conjecture to be necessary.
\end{abstract}

\section{INTRODUCTION}

In [12] (see also [10]) Moon and Rand introduced the following system of differential equations, which we shall call the Moon-Rand system, in the context of modelling control of flexible structures:

$$
\begin{aligned}
\dot{u} & =v \\
\dot{v} & =-u-u w \\
\dot{w} & =-\lambda w+f(u, v)
\end{aligned}
$$

where

$$
f(u, v)=c_{20} u^{2}+c_{11} u v+c_{02} v^{2} \quad \text { or } \quad f(u, v)=\frac{c_{11} u v}{1+\eta u^{2}} .
$$

Here $\lambda, \eta, c_{20}, c_{11}$ and $c_{02}$ are real numbers, $\lambda>0, \eta>0$. They showed that in the former (polynomial feedback) case the origin is asymptotically

2010 Mathematics Subject Classification. 34C05 34A34.

Key words and phrases. Center problem, cyclicity, integrability, stability.

The research of the second author was supported by the Slovenian Research Agency. The authors also acknowledge the support of Slovenia-USA bilateral grant BI-US/09-12-09. 
stable for the flow restricted to the center manifold if

$$
2 c_{20}-2 c_{02}-\lambda c_{11}<0 .
$$

This condition was found by approximating the local center manifold $W^{c}$ of (1.1), transforming the system restricted to $W^{c}$ to a normal form by means of an unspecified near-identity transformation, and going over to polar coordinates.

In this paper we give a complete stability analysis of the flow restricted to a neighborhood of the origin in any center manifold. We allow arbitrary values of $\eta$ and negative values of $\lambda$, requiring only that $\lambda$ be nonzero so that the singularity at the origin be isolated. For the flow on any center manifold the origin is either a center or a fine focus of order up to three in the polynomial case and four in the rational case. We derive discriminant quantities which specify the order and stability of foci. This is done by approximating any local center manifold $W^{c}$ at the origin and computing the first few Lyapunov or Poincaré-Lyapunov quantities (see Section 2), which also permits us to show that a fine focus of order $k$ can be made to bifurcate $k-1$ limit cycles under small perturbation within the family (1.1), and a center either one or two limit cycles in the polynomial case and one, two, or three limit cycles in the rational case. This implies existence of open sets of parameter values for which the system possesses both an asymptotically stable equilibrium and an asymptotically stable periodic orbit. An interesting byproduct of the analysis is that the sign of the normal contraction factor $\lambda$ affects the asymptotic stability of the origin as a fixed point of the flow restricted to the center manifold.

The approximation process described in the previous paragraph will not do for the center problem; in general power series expansions for center manifolds of analytic or even polynomial systems need not converge (see [1] and [14]). Instead we avoid the center manifold entirely using the ideas developed in [8] (and reviewed in Section 2): complexify (1.1) and compute a sufficiently long initial string of an infinite sequence of polynomials $\widetilde{g}_{k}$ in the ring $\mathbb{C}\left[\lambda, c_{20}, c_{11}, c_{02}\right]$, the focus quantities of family (1.1), whose variety specifies those systems in (1.1) with a center at the origin on the center manifold (which is unique in such circumstances). By this means we are able to solve the center problem for the flow of (1.1) on the center manifold. We also derive the curious result that (for both polynomial and rational 
feedback) there is a center at the origin in the center manifold only if the center manifold is an algebraic surface.

In the polynomial case we generalize system (1.1) in a natural way to the generalized polynomial Moon-Rand system:

$$
\begin{aligned}
\dot{u} & =v \\
\dot{v} & =-u-u w \\
\dot{w} & =-\lambda w+\sum_{j+k=n} c_{j k} u^{j} v^{k} .
\end{aligned}
$$

As is typical of three-dimensional systems, even with just a few parameters computations quickly become intractable, even for special-purpose computer algebra systems. The parameter $\lambda$ causes the greatest difficulty. By fixing its value we are able to solve the center problem for family (1.2) for $n=3$, but only by computing using modular arithmetic, and that in a somewhat novel way.

In the general case we produce conditions that guarantee existence of a center on the center manifold of (1.2), which is then algebraic, and offer evidence in support of a conjecture that these conditions are necessary, which implies that the center manifold is always algebraic if it contains a center. Finally, we generalize the result of Moon and Rand to this situation by deriving a sufficient condition for asymptotic stability of the origin for the flow restricted to the center manifold.

In the following section we review background facts. Section 3 is devoted to the polynomial Moon-Rand system and Section 4 to the rational MoonRand system (1.1). Of course the polynomial family is the special case $\eta=0$ of the rational family, but we have separated out the treatment of this case for several reasons. The results for the two families are similar but enough different that a separate statements of the results is much more readable. We use the two separate theorems to illustrate two approaches to the proof of the results. The use of the Lyapunov function approach in proving Theorem 4.1 is computationally efficient, but the polar coordinate approach in proving Theorem 3.2 makes one observation more transparent, and facilitates the proof of the cyclicity theorems, Theorems 3.5 and 4.2.

In Section 5 we study the center and stability problems for the generalized polynomial Moon-Rand system (1.2). 


\section{The Focus Quantities and the Center Variety}

Consider a three dimensional system that can be placed in the form

$$
\begin{aligned}
\dot{u} & =-v+P(u, v, w)=\widetilde{P}(u, v, w) \\
\dot{v} & =\quad u+Q(u, v, w)=\widetilde{Q}(u, v, w) \\
\dot{w}=-\lambda w+R(u, v, w) & =\widetilde{R}(u, v, w),
\end{aligned}
$$

where $\lambda$ is a non-zero real number and $P, Q$, and $R$ are polynomial functions without constant or linear terms. We will let $\mathfrak{X}$ denote the corresponding vector field

$$
\mathfrak{X}=\widetilde{P} \frac{\partial}{\partial u}+\widetilde{Q} \frac{\partial}{\partial v}+\widetilde{R} \frac{\partial}{\partial w}
$$

on a neighborhood of the origin.

A proof of the following theorem can be found in [8]. The equivalence of statements (a) and (b) is called the Lyapunov Center Theorem; it is proved in many places, including [4].

Theorem 2.1. Fix a system (2.1) in which the functions $P, Q$, and $R$ are real analytic on a neighborhood of the origin and let $W^{c}$ be the local center manifold at the origin. The following statements are equivalent.

(a) The origin is a center for $\mathfrak{X} \mid W^{c}$.

(b) System (2.1) admits a local analytic first integral.

(c) System (2.1) admits a formal first integral.

In fact a real analytic local first integral from statement (b) can always be chosen to be of the form

$$
\Phi(u, v, w)=u^{2}+v^{2}+\cdots
$$

in a neighborhood of the origin in $\mathbb{R}^{3}$.

Introducing the complex variable $x=u+i v$, the first two equations in (2.1) are equivalent to a single equation $\dot{x}=i x+X(x, \bar{x}, w)$, where $X$ is a sum of homogeneous polynomials of degrees between two and $N=$ $\max (\operatorname{deg} P, \operatorname{deg} Q, \operatorname{deg} R)$. Adjoining to this equation its complex conjugate, replacing $\bar{x}$ everywhere by $y$, regarding $y$ as an independent complex variable, and replacing $w$ by $z$ simply as a notational convenience we obtain the 
complexification of family (2.1),

$$
\begin{aligned}
& \dot{x}=i x+\sum_{p+q+r=2}^{N} a_{p q r} x^{p} y^{q} z^{r} \\
& \dot{y}=-i y+\sum_{p+q+r=2}^{N} b_{p q r} x^{p} y^{q} z^{r} \\
& \dot{z}=-\lambda z+\sum_{p+q+r=2}^{N} c_{p q r} x^{p} y^{q} z^{r},
\end{aligned}
$$

where $b_{q p r}=\bar{a}_{p q r}$ and the $c_{p q r}$ are such that $\sum_{p+q+r=2}^{N} c_{p q r} x^{p} \bar{x}^{q} w^{r}$ is real for all $x \in \mathbb{C}$, for all $w \in \mathbb{R}$. Let $\mathfrak{Z}$ denote the corresponding vector field on $\mathbb{C}^{3}$. Existence of a first integral $\Phi(u, v, w)=u^{2}+v^{2}+\cdots$ for a system in family (2.1) is equivalent to existence of a first integral

$$
\Psi(x, y, z)=x y+\sum_{j+k+\ell=3} v_{j k \ell} x^{j} y^{k} z^{\ell}
$$

for the corresponding system in family (2.3).

When $\Psi$ has the form (2.4) the coefficient $g_{k_{1} k_{2} k_{3}}$ of $x^{k_{1}} y^{k_{2}} z^{k_{3}}$ in $\mathfrak{Z} \Psi$ can be calculated explicitly (see [8]). Except when $\left(k_{1}, k_{2}, k_{3}\right)=(K, K, 0)$ for $K \in \mathbb{N}$, the equation $g_{k_{1} k_{2} k_{3}}=0$ can be solved uniquely for $v_{k_{1} k_{2} k_{3}}$ in terms of the known quantities $v_{\alpha \beta \gamma}$. A formal first integral $\Psi$ thus exists if $g_{k k 0}=0$ for all $k \in \mathbb{N}$. An obstruction to the existence of the formal series $\Psi$ occurs when the coefficient $g_{K K 0}$ is nonzero. This coefficient is the $K$ th focus quantity.

The focus quantities $g_{110}$ and $g_{220}$ are uniquely determined, but the remaining ones depend on the choices made for $v_{K K 0}, K \in \mathbb{N}, K \geq 2$. Once such an assignment is made $\Psi$ is determined and satisfies

$$
\mathfrak{Z} \Psi(x, y, z)=g_{110} x y+g_{220}(x y)^{2}+g_{330}(x y)^{3}+\cdots .
$$

Vanishing of all the focus quantities is sufficient for existence of the formal first integral. It is known ([8]) that it is necessary by proving that if for one choice of the $v_{K K 0}$ at least one focus quantity is non-zero, then the same is true for every other choice of the $v_{K K 0}$. To shorten the notation we let $(a, b, c)$ stand for the coefficient string

$$
\left(a_{200}, \ldots, a_{00 N}, b_{200}, \ldots, b_{00 N}, c_{200}, \ldots, c_{00 N}\right) .
$$

Theorem 2.2. Let $\Psi$ be a formal series of the form (2.4) and let $g_{110}, g_{220}, \ldots$ be polynomials in $(a, b, c)$ that satisfy (2.5) with respect to the system (2.3). 
Then system (2.3) with $(a, b, c)=\left(a^{*}, b^{*}, c^{*}\right)$ admits a formal first integral of the form (2.4) if and only if $g_{k k 0}\left(a^{*}, b^{*}, c^{*}\right)=0$ for all $k \in \mathbb{N}$.

The following theorem shows that the conditions for the existence of a center on the local center manifold of a polynomial vector field in $\mathbb{R}^{3}$ can always be expressed as zeros of polynomials. For a proof see [8]. Recall that the set of common zeros of a collection of polynomials that generate an ideal $I$ is the variety $\mathbf{V}(I)$ of $I$.

Theorem 2.3. Let (2.1) be a family of polynomial differential equations on $\mathbb{R}^{3}$. For any system in the family let $\mathfrak{X}$ be the corresponding vector field (2.2) and let $W^{c}$ be a local center manifold through the origin. Then there exists a variety $V_{C}$ in the space of admissible coefficients such that the origin is a center for $\mathfrak{X} \mid W^{c}$ if and only if the coefficients of the components of $\mathfrak{X}$ lie in $V_{C}$.

If $\mathfrak{X}=\sum P_{j} \partial / \partial u_{j}$ is a polynomial vector field of degree $m$ on $\mathbb{R}^{n}$ then the variety of a polynomial $F$, the algebraic hypersurface $F=0$ in $\mathbb{R}^{n}$, is invariant under the flow induced by $\mathfrak{X}$ if there exists a polynomial $K$, which can have degree up to $m-1$, such that

$$
\mathfrak{X} F-K F=\sum P_{j} \frac{\partial F}{\partial u_{j}}-K F=0 .
$$

(The converse requires that the ground field be $\mathbb{C}$; see $[13, \S 3.6]$.)

For a system of differential equations on $\mathbb{R}^{2}$ of the form

$$
\dot{u}=-v+P(u, v), \quad \dot{v}=u+Q(u, v)
$$

where $P$ and $Q$ are sufficiently smooth and vanish together with their first partial derivatives let $\mathcal{R}(r)$ denote the Poincaré first return map on a sufficiently short segment of the polar axis and $\mathcal{D}$ the difference map $\mathcal{D}(r)=\mathcal{R}(r)-r$. There always exists a sufficiently smooth function $V$ from a neighborhood of the origin into $\mathbb{R}$ of the form $V(u, v)=\left(u^{2}+v^{2}\right) / 2+\cdots$ such $\mathfrak{X} V=L_{4}\left(u^{2}+v^{2}\right)^{2}+L_{6}\left(u^{2}+v^{2}\right)^{3}+\cdots$. By the $k$ th Lyapunov quantity we mean the coefficient $\eta_{k}$ in the expansion $\mathcal{D}(r)=\eta_{1} r+\eta_{2} r^{2}+\cdots$. By the $k$ th Poincaré-Lyapunov quantity we mean the coefficient $L_{2 k}$.

\section{The Polynomial Moon-Rand System}

In Theorem 3.2 we present a full characterization of the stability of the origin of the Moon-Rand system as well as a determination of the center conditions on the center manifold. We begin with a computation of the 
lowest order terms of any center manifold. The first three were already found in [12]. The higher order coefficients will be needed only after simplifying conditions apply, so only the simpler versions are listed. See also Lemma 5.6 below.

Lemma 3.1. Let a center manifold at the origin of the Moon-Rand system (1.1) with polynomial $f$ be expressed as

$$
w=h(u, v)=p_{20} u^{2}+p_{11} u v+p_{02} v^{2}+\cdots .
$$

Then $p_{j k}=0$ if $j+k$ is odd. In general

$$
\begin{aligned}
& p_{20}=\frac{1}{\lambda\left(\lambda^{2}+4\right)}\left(2 c_{02}+2 c_{20}+\lambda c_{11}+\lambda^{2} c_{20}\right) \\
& p_{11}=\frac{1}{\left(\lambda^{2}+4\right)}\left(2 c_{02}-2 c_{20}+\lambda c_{11}\right) \\
& p_{02}=\frac{1}{\lambda\left(\lambda^{2}+4\right)}\left(2 c_{02}+2 c_{20}-\lambda c_{11}+\lambda^{2} c_{02}\right) .
\end{aligned}
$$

When $c_{02}=c_{20}-(\lambda / 2) c_{11}$

$$
\begin{aligned}
& p_{13}=\frac{1}{2 \lambda\left(\lambda^{2}+4\right)\left(\lambda^{2}+16\right)}\left(\lambda c_{11}-2 c_{20}\right)\left(\lambda\left(\lambda^{2}+10\right) c_{11}-2\left(\lambda^{2}+16\right) c_{20}\right) \\
& p_{31}=\frac{1}{\lambda\left(\lambda^{2}+4\right)\left(\lambda^{2}+16\right)}\left(\lambda c_{11}-2 c_{20}\right)\left(3 \lambda c_{11}-\left(\lambda^{2}+16\right) c_{20}\right) .
\end{aligned}
$$

When $c_{20}=(\lambda / 4) c_{11}$ and $c_{02}=c_{20}-(\lambda / 2) c_{11}=-(\lambda / 4) c_{11}$

$$
\begin{aligned}
& p_{51}=\frac{\lambda\left(2 \lambda^{4}+53 \lambda^{2}+216\right)}{2\left(\lambda^{2}+16\right)^{2}\left(\lambda^{4}+40 \lambda^{2}+144\right)} c_{11}^{3} \\
& p_{33}=-\frac{3 \lambda\left(3 \lambda^{2}+8\right)}{4\left(\lambda^{2}+16\right)\left(\lambda^{4}+40 \lambda^{2}+144\right)} c_{11}^{3} \\
& p_{15}=\frac{\lambda\left(\lambda^{4}+13 \lambda^{2}+72\right)}{2\left(\lambda^{2}+16\right)^{2}\left(\lambda^{4}+40 \lambda^{2}+144\right)} c_{11}^{3} .
\end{aligned}
$$

Proof. The coefficients $p_{j k}$ are found by equating coefficients in the expression that determines the center manifold,

$$
h_{u} \dot{u}+h_{v} \dot{v}=-\lambda h+c_{20} u^{2}+c_{11} u v+c_{02} v^{2} .
$$

When this expression is written out with homogeneous terms in $h$ collected the assertion that $p_{j k}=0$ if $j+k$ is odd follows by induction and Lemma 5.5 . 
The first three coefficients listed in the present lemma can be found manually, but the computer algebra system Mathematica was used for the remaining ones.

Theorem 3.2. Let $\mathfrak{X}$ denote the vector field determined on $\mathbb{R}^{3}$ by the MoonRand system (1.1) with polynomial $f$. Define the discriminant quantities

$$
\begin{aligned}
& W_{1}=2 c_{20}-2 c_{02}-\lambda c_{11} \\
& W_{2}=-\lambda\left(2 c_{20}-\lambda c_{11}\right)\left(4 c_{20}-\lambda c_{11}\right) \\
& W_{3}=-\lambda c_{02}^{2} c_{11}
\end{aligned}
$$

For any center manifold $W^{c}$ of (1.1) at the origin of $\mathbb{R}^{3}$, with regard to $\mathfrak{X} \mid W^{c}$ :

a. if $W_{1} \neq 0$ then the origin is a first order fine focus whose stability is determined by $\operatorname{sgn} W_{1}$ (i.e., is asymptotically stable iff $W_{1}<0$ );

b. if $W_{1}=0$ but $W_{2} \neq 0$ then the origin is a second order fine focus whose stability is determined by $\operatorname{sgn} W_{2}$;

c. if $W_{1}=W_{2}=0$ but $W_{3} \neq 0$ then the origin is a third order fine focus whose stability is determined by $\operatorname{sgn} W_{3}$;

d. if $W_{1}=W_{2}=W_{3}=0$ then the origin is a center;

e. the origin is a center iff $c_{02}=2 c_{20}-\lambda c_{11}=0$.

Proof. We prove part (e) first. The first four nonzero focus quantities were computed by means of the method described in Section 2, first complexifying (1.1) and then computing as described earlier. The first two nonzero focus quantities, for example, are

$$
\begin{aligned}
& g_{220}=\frac{2 c_{20}-2 c_{02}-c_{11} \lambda}{4+\lambda^{2}}, \\
& g_{330}=\frac{\left(c_{20}+c_{02}\right)\left[c_{11} \lambda\left(12+\lambda^{2}\right)-2 c_{02}\left(-4+\lambda^{2}\right)-2 c_{20}\left(12+\lambda^{2}\right)\right]}{4 \lambda\left(4+\lambda^{2}\right)^{2}} .
\end{aligned}
$$

All were computed using Mathematica.

Let $\widetilde{g}_{k k 0}$ denote the numerator of $g_{k k 0}$ and $\mathcal{B}_{5}=\left\langle\widetilde{g}_{220}, \widetilde{g}_{330}, \widetilde{g}_{440}, \widetilde{g}_{550}\right\rangle$. Using the special-purpose computer algebra system Singular ([7], [9]) to decompose the radical of $\mathcal{B}_{5}$ into a unique intersection of prime ideals, we obtain the irreducible decomposition of the variety $\mathbf{V}\left(\mathcal{B}_{5}\right)$ as the union of three components $\mathbf{V}\left(J_{j}\right)$, where the ideals $J_{j}$ are:

$$
\begin{aligned}
& J_{1}=\left\langle c_{02},-\lambda c_{11}+2 c_{20}-2 c_{02}\right\rangle \\
& J_{2}=\left\langle c_{11}^{2}+16 c_{02}^{2}, 4 \lambda c_{02}-c_{11}, \lambda c_{11}+4 c_{02}, \lambda^{2}+1,-\lambda c_{11}+2 c_{20}-2 c_{02}\right\rangle \\
& J_{3}=\left\langle\lambda^{2}+4,-\lambda c_{11}+2 c_{20}-2 c_{02}\right\rangle .
\end{aligned}
$$


Since system (1.1) is real the components $\mathbf{V}\left(J_{2}\right)$ and $\mathbf{V}\left(J_{3}\right)$ are irrelevant; we get the necessary conditions

$$
c_{02}=2 c_{20}-\lambda c_{11}=0
$$

for the origin to be a center for $\mathfrak{X} \mid W^{c}$.

When these conditions hold it is not too difficult to find the algebraic surface

$$
F(u, v, w):=c_{20} u^{2}-\lambda w=0
$$

as an invariant surface (with cofactor $-\lambda$ ) for the flow associated to system (1.1). Since it is tangent to the plane $w=0$ at the origin it is in fact a center manifold for this system. Using this explicit expression for a center manifold $W^{c}$ we find that the dynamics on $W^{c}$ are given by

$$
\begin{aligned}
& \dot{u}=v \\
& \dot{v}=-u+\frac{c_{20}}{\lambda} u^{3} .
\end{aligned}
$$

This system is Hamiltonian with the Hamiltonian function

$$
H(u, v)=\frac{1}{2}\left(u^{2}+v^{2}\right)+\frac{c_{20}}{4 \lambda} u^{4}
$$

so it admits a center at the origin. Thus the condition in part (e) is also sufficient, and part (e) is established.

Now we turn to parts (a) through (d), in order. The system $\mathfrak{X} \mid W^{c}$ is

$$
\begin{aligned}
& \dot{u}=v \\
& \dot{v}=-u-u h(u, v)
\end{aligned}
$$

for $h(u, v)=\sum p_{j k} u^{j} v^{k}$ whose first few coefficients are given in Lemma 3.1. We find the first few Lyapunov quantities in the usual way. (The approach to stability using the Poincaré-Lyapunov quantities based on Lyapunov functions, which can be computationally simpler, could have been used. See the proof of Theorem 4.1.) Using the notation in Section 3.1 of [13], in polar coordinates system (3.4) is

$$
\begin{aligned}
& \dot{r}=-r \cos \theta \sin \theta h(r \cos \theta, r \sin \theta)=-\sum \alpha_{j} r^{j} \\
& \dot{\theta}=-1-\cos ^{2} \theta h(r \cos \theta, r \sin \theta)=-1-\sum \beta_{j} r^{j}
\end{aligned}
$$

where

$$
\begin{aligned}
& \alpha_{j}(\theta)=\cos \theta \sin \theta\left(\sum_{k+\ell=j-1} p_{k \ell} \cos ^{k} \theta \sin ^{\ell} \theta\right), \quad j \geq 3 \\
& \beta_{j}(\theta)=\cos ^{2} \theta\left(\sum_{k+\ell=j} p_{k \ell} \cos ^{k} \theta \sin ^{\ell} \theta\right), \quad j \geq 2 .
\end{aligned}
$$


Since $p_{k \ell}=0$ when $k+\ell$ is odd, $\alpha_{2 n}=\beta_{2 n+1}=0$. Then

$$
\frac{d r}{d \theta}=\sum R_{j}(\theta) r^{j}
$$

where

$$
\begin{array}{lcrl}
R_{1}=0 & R_{2}=0 & R_{3} & =\alpha_{3} \\
R_{4}=0 & R_{5}=\alpha_{5}-\alpha_{3} \beta_{2} & R_{6} & =0 \\
R_{7}=\alpha_{7}-\alpha_{5} \beta_{2}-\alpha_{3} \beta_{4}+\alpha_{3} \beta_{2}^{2} & R_{8} & =0 \\
R_{9}=\alpha_{9}-\alpha_{7} \beta_{2}+\alpha_{5} \beta_{2}^{2}-\alpha_{3} \beta_{2}^{3}-\alpha_{5} \beta_{4} & +2 \alpha_{3} \beta_{2} \beta_{4}-\alpha_{3} \beta_{6}
\end{array}
$$

It is important to note that since $\dot{\theta}<0$ near 0 (arising from the noncanonical location of the minus sign in the linear part of (1.1) that gives rise to the complex eigenvalues; compare with (2.7)), in passing from (3.5) to (3.7) the direction of time is essentially reversed: as $\theta$ increases from 0 to $2 \pi$ time $t$ decreases. Therefore the usual polar coordinate procedure for computing the Lyapunov quantities gives the negatives of the correct values. The negative of the first Lyapunov quantity, $-\eta_{1}$, is $w_{1}(2 \pi)-1$ and for $j \geq 2$ the negative of the $j$ th Lyapunov quantity, $-\eta_{j}$, is $w_{j}(2 \pi)$, where the $w_{j}$ are the solutions of the differential equations that arise from

$$
\sum w_{j}^{\prime}(\theta) r_{0}^{j}=\sum R_{j}(\theta)\left(w_{1}(\theta) r_{0}+w_{2}(\theta) r_{0}^{2}+\cdots\right)^{j}
$$

with initial values

$$
w_{1}(0)=1, \quad w_{j}(0)=0 \quad \text { for } \quad j>1 .
$$

In particular,

$$
w_{1}^{\prime}=R_{1} w_{1}=0, \quad w_{1}(0)=1
$$

yields $w_{1}(\theta) \equiv 1$, so that $\eta_{1}=0$, and

$$
w_{2}^{\prime}=R_{2} w_{1}^{2}=0, \quad w_{2}(0)=0
$$

yields $w_{2}(\theta) \equiv 0$, so that $\eta_{2}=0$. Then

$$
w_{3}^{\prime}=R_{3}=\alpha_{3}, \quad w_{3}(0)=0
$$

yields

$$
w_{3}(\theta)=\frac{1}{4} p_{20}+\frac{1}{8} p_{11} \theta-\frac{1}{4} p_{20} \cos ^{4} \theta+\frac{1}{4} p_{02} \sin ^{4} \theta-\frac{1}{32} p_{11} \sin 4 \theta
$$

so that

$$
\eta_{3} \sim-p_{11} \sim 2 c_{20}-2 c_{02}-\lambda c_{11}
$$


where $a \sim b$ means that $a$ is a positive constant times $b$. This establishes point (a).

From (3.8) $w_{4}^{\prime}=0$ so $w_{4} \equiv 0$, hence $\eta_{4} \equiv 0$. It is apparent that $w_{4}=0$ in turn implies that $w_{6}^{\prime}=0$ so $w_{6} \equiv 0$, hence $\eta_{6} \equiv 0$ (and $w_{4}=w_{6}=0$ imply that $w_{8}^{\prime}=0$ so $w_{8} \equiv 0$, hence $\left.\eta_{8} \equiv 0\right)$.

From (3.8)

$$
w_{5}^{\prime}=3 R_{3} w_{1}\left(w_{2}^{2}+w_{1} w_{3}\right)+4 R_{4} w_{1}^{3} w_{2}+R_{5} w_{1}^{5}=3 R_{3} w_{3}+R_{5} .
$$

At this point hand computations are practically infeasible and intermediate results too long to copy here. Proceeding on the assumption that $\eta_{3}=0$, so that $p_{11}=0$, which we implement by the substitution $c_{02}=c_{20}-(\lambda / 2) c_{11}$, we use the symbolic manipulator Mathematica to compute $w_{5}$ and obtain $\eta_{5} \sim p_{31}+p_{13}$, which by (3.2) gives

$$
\eta_{5}=-\frac{\left(2 c_{20}-\lambda c_{11}\right)\left(4 c_{20}-\lambda c_{11}\right)}{2 \lambda\left(4+\lambda^{2}\right)}
$$

which has the sign of $W_{2}$. Since $\eta_{4} \equiv 0$ (or appealing to the fact that the first non-zero Lyapunov quantity has odd index) this establishes part (b).

If $\eta_{5}=0$ because $2 c_{20}-\lambda c_{11}=0$ then $\eta_{3}=0$ implies that $c_{02}=0$ as well, and by part (e) of the theorem the origin is a center. The factor $c_{02}^{2}$ in $W_{3}$ makes $W_{3}=0$ in this case. We proceed on the assumption that $\eta_{5}=0$ but $2 c_{20}-\lambda c_{11} \neq 0$. Thus $4 c_{20}-\lambda c_{11}=0$ and (from $\eta_{3}=0$ ) $c_{02}=c_{20}-(\lambda / 2) c_{11} \neq 0$. From (3.8) and what we already know we have

$$
w_{7}^{\prime}=3 R_{3}\left(w_{3}^{2}+w_{5}\right)+4 R_{4} w_{4}+5 R_{5} w_{3}+R_{7}
$$

which with the initial condition gives

$$
\eta_{7}=w_{7}(2 \pi) \sim-5 p_{15}-5 p_{51}-3 p_{33}+p_{02} p_{31}+5 p_{20} p_{31} \sim-\lambda c_{11}^{3} .
$$

Since $c_{02} \neq 0, \eta_{7}$ is zero iff $W_{3}=-\lambda c_{02}^{2} c_{11}$ is zero, and has the same sign as $W_{3}$ when they are nonzero. Since $\eta_{6} \equiv 0$ this establishes (c). (In fact, in the case at hand $\eta_{7} \neq 0$, for since we have assumed $W_{1}=W_{2}=0, c_{11}=0$ would ultimately imply that $c_{02}=0$. The reader is reminded that what is shown in the last display is not $\eta_{7}$, but $\eta_{7}$ under certain restrictive conditions.) Recalling the comment above that if $\eta_{5}$ vanishes because $2 c_{20}-\lambda c_{11}=0$ then $W_{3}=0$ is forced, point (d) holds as well.

Figure 1 shows, for any fixed value of $\lambda$, the decomposition of the parameter space according to the stability of the origin of the induced system on any center manifold. Off the plane $\Pi: 2 c_{20}-\lambda c_{11}-2 c_{02}=0$ (which is never 
the plane $c_{02}=0$ ) the origin is a first order fine focus. Within $\Pi$ but off the lines

$$
\begin{array}{r}
\ell: \Pi \cap\left\{\left(c_{20}, c_{11}, c_{02}\right): 4 c_{20}-\lambda c_{11}=0\right\} \\
\mu: \Pi \cap\left\{\left(c_{20}, c_{11}, c_{02}\right): 2 c_{20}-\lambda c_{11}=0\right\}
\end{array}
$$

(which are always in general position) the origin is a second order fine focus. Along the line $\ell$ but away from its intersection with the line $\mu$ the origin is a third order fine focus. The center variety is the line $\mu$.

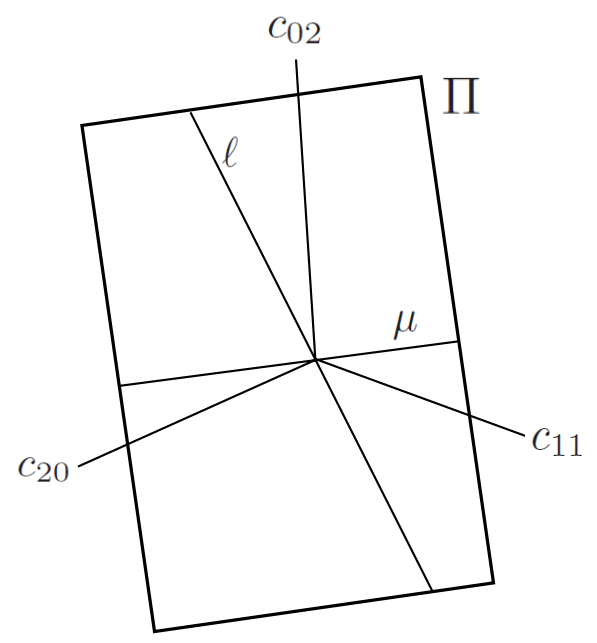

Figure 1. Decomposition of the parameter space of system (1.1) according to stability of the origin.

Remark 3.3. We note the interesting fact that although the parameter $\lambda$ appears only in as the rate of linear expansion or contraction normal to the center eigenspace, when the focus in $W^{c}$ is of order two or greater its asymptotic stability depends on the sign of $\lambda$. Theorem 4.1 shows that the same fact holds for the case of rational feedback.

Remark 3.4. In the proof of the theorem we saw that if system (1.1) has a center on a center manifold at the origin then the unique center manifold is an algebraic surface. We will see the same result for rational feedback, and suspect that it is the case in general. See Conjecture 5.3 and Proposition 5.4 . 
Since the first non-zero Lyapunov quantity is known under every circumstance, bifurcation of limit cycles from the origin can also be discussed. Uncertainty as to the analyticity of the center manifold prevents us from being able to assert sharpness of the bounds, hence the cyclicity of the singularity, in the case of a center. On the other hand, there always exists a local center manifold of arbitrarily high smoothness ([14]), and the flows on any two $C^{k+1}$ center manifolds are $C^{k}$ conjugate on a neighborhood of the origin ([3]) so that all contain the same number of small cycles. Thus the statements in the following theorem do not depend on the center manifold selected, if there is more than one. We also remark that, even though center manifolds may not be analytic, if there are infinitely many cycles in a neighborhood of the origin then the origin is a center ([1]).

Theorem 3.5. For family (1.1) with polynomial $f$ restrict attention to the flow on a center manifold at the origin.

a. A first order fine focus at the origin has cyclicity zero: no limit cycles bifurcate from it under small perturbation within (1.1).

b. For $k=2$ and $k=3$, a fine focus of order $k$ at the origin has cyclicity $k-1$ : up to $k-1$ limit cycles can be made to bifurcate under small perturbation within the family (1.1).

c. In the case $c_{20}=c_{11}=c_{02}=0$ the center on the center manifold $w=0$ can be made to bifurcate two limit cycles. Otherwise the center on the center manifold can be made to bifurcate one limit cycle.

Proof. A first order fine focus at the origin has cyclicity zero because $\eta_{1}$ is always zero.

An upper bound of $k$ on the cyclicity of a $k$ th order fine focus can be obtained directly from an application of the finitely differentiable version of the Weierstrass-Malgrange Preparation Theorem ([11]). To obtain a sharp bound we imitate the argument in the proof of Proposition 6.1.2 of [13] (see also [2]).

To abbreviate the notation write $\xi=\left(\lambda, c_{20}, c_{11}, c_{02}\right) \in \mathbb{R}^{4}$ for the parameters. To obtain an upper bound on the number of limit cycles that can bifurcate from a fine focus of order two or three let us suppose to be specific that for some $\xi^{*}$ the origin is a fine focus of order three and consider the system restricted to a center manifold that is $C^{r}$ for $r \geq 8$. Fix a neighborhood $N$ of $\xi^{*}$ on which $\eta_{7}=\eta_{7}(\xi) \neq 0$ and an interval $I=[0, \epsilon)$ so that the difference map $\mathcal{D}=\mathcal{D}(r, \xi)$ is defined on $I \times N$. Then since as seen in the 
proof of Theorem $3.2 \eta_{4}$ and $\eta_{6}$ are identically zero

$$
\mathcal{D}(r, \xi)=\eta_{3}(\xi) r^{3}+\eta_{5}(\xi) r^{5}+\eta_{7}(\xi) r^{7}+R(r, \xi)
$$

where $R$ is at least $C^{7}$ and $R^{(j)}(0, \xi)=0$ for $0 \leq j \leq 7$.

Divide $\mathcal{D}$ by $r^{3}$ (defined at zero by the limit) and differentiate with respect to $r$ to obtain the function

$$
\mathcal{D}_{1}(r, \xi)=2 \eta_{5}(\xi) r+4 \eta_{7}(\xi) r^{3}+R_{1}(r, \xi)
$$

where $R_{1}$ is at least $C^{3}$ and $R_{1}^{(j)}(0, \xi)=0$ for $0 \leq j \leq 3$. Either $\mathcal{D}$ and $\mathcal{D}_{1}$ both have infinitely many zeros on $(0, \epsilon)$ or $\mathcal{D}$ has at most one more zero on $(0, \epsilon)$ than $\mathcal{D}$ does.

Divide $\mathcal{D}_{1}$ by $r$ (defined at zero by the limit) and differentiate with respect to $r$ to obtain the function

$$
\mathcal{D}_{2}(r, \xi)=8 \eta_{7}(\xi) r+R_{2}(r, \xi)
$$

where $R_{2}$ is at least $C^{1}$ and $R_{2}^{(j)}(0, \xi)=0$ for $0 \leq j \leq 1$. Either $\mathcal{D}_{1}$ and $\mathcal{D}_{2}$ both have infinitely many zeros on $(0, \epsilon)$ or $\mathcal{D}_{1}$ has at most one more zero on $(0, \epsilon)$ than $\mathcal{D}_{2}$ does.

Divide $\mathcal{D}_{2}$ by $r$ (defined at zero by the limit) to obtain the function

$$
\mathcal{D}_{3}(r, \xi)=8 \eta_{7}(\xi)+R_{3}(r, \xi)
$$

where $R_{3}$ is continuous and $R_{3}(0, \xi)=0$. For $\xi$ in a neighborhood $N^{\prime} \subset N$ of $\xi^{*}$ and an $\epsilon^{\prime} \in(0, \epsilon), \mathcal{D}_{3}(r, \xi)$ has no zeros in $\left(0, \epsilon^{\prime}\right)$. Thus $\mathcal{D}(r, \xi)$ has at most two zeros in $\left(0, \epsilon^{\prime}\right)$ for all $\xi \in N^{\prime}$.

In the same way a second order fine focus can be made to bifurcate at most one limit cycle.

The bounds are sharp because, as shown in the proof of Theorem 3.2, the Lyapunov quantities $\eta_{3}, \eta_{5}$, and $\eta_{7}$ can be adjusted independently. To be specific, suppose the origin is a third order fine focus, so that $\eta_{j}=0$ for $1 \leq j \leq 6$ but $\eta_{7} \neq 0$. We must have $4 c_{20}-\lambda c_{11}=0$ but $2 c_{20}-\lambda c_{11} \neq 0$ else by $\eta_{3}=0, c_{02}=0$ is forced and the singularity is a center. Moving $c_{11}$ by an arbitrarily small amount in the correct direction, leaving $c_{20}$ unchanged, but maintaining $c_{02}=c_{20}-\lambda c_{11} / 2$ makes the sign of $\eta_{5}$ change to the opposite sign of that of $\eta_{7}$ but maintains $\eta_{3}=0$. A zero of the difference map on a section of the flow near the origin is produced, corresponding to a limit cycle. Then $c_{20}$ or $c_{02}$ can be moved by an arbitrarily small amount to create a second limit cycle. A single limit cycle can be produced similarly from a second order fine focus. 
If the origin is a center, then unless $c_{20}=c_{11}=c_{02}=0$ there is no third order fine focus near it and the same technique produces one limit cycle. When $c_{20}=c_{11}=c_{02}=0$ one can first make an arbitrarily small perturbation to a third order fine focus, and from there produce two limit cycles.

\section{The Rational Moon-Rand System}

In this section we perform the same analysis for the rational Moon-Rand system that was done for the polynomial Moon-Rand system in Section 3. We shall consider the following slight generalization of the rational MoonRand system (1.1):

$$
\begin{aligned}
\dot{u} & =v \\
\dot{v} & =-u-u w \\
\dot{w} & =-\lambda w+f(u, v), \quad f(u, v)=\frac{c_{20} u^{2}+c_{11} u v+c_{02} v^{2}}{1+\eta u^{2}}
\end{aligned}
$$

where $c_{20}, c_{11}, c_{02}, \lambda$, and $\eta$ are real parameters. Of course for $\eta=0$ this is just the polynomial system.

Theorem 4.1. Let $\mathfrak{X}$ denote the vector field determined on $\mathbb{R}^{3}$ by the rational Moon-Rand system (4.1). Define the discriminant quantities

$$
\begin{aligned}
& W_{1}=2 c_{20}-2 c_{02}-\lambda c_{11} \\
& W_{2}=-\lambda\left(4 c_{20}-\lambda c_{11}\right)\left(2 c_{20}-\lambda c_{11}+2 \eta \lambda\right) \\
& W_{3}=-\lambda c_{11}\left(c_{11}-4 \eta\right)\left[\left(\lambda^{2}+10\right) c_{11}+64 \eta+4 \lambda^{2} \eta\right]\left(2 c_{20}-\lambda c_{11}+2 \eta \lambda\right)^{2} \\
& W_{4}=\lambda \eta^{2} c_{11}^{2}\left(c_{11}-4 \eta\right)^{2}\left(2 c_{20}-\lambda c_{11}+2 \eta \lambda\right)^{2}
\end{aligned}
$$

For any center manifold $W^{c}$ of (4.1) at the origin of $\mathbb{R}^{3}$, with regard to $\mathfrak{X} \mid W^{c}$ :

a. if $W_{1} \neq 0$ then the origin is a first order fine focus whose stability is determined by $\operatorname{sgn} W_{1}$ (i.e., is asymptotically stable iff $W_{1}<0$ );

b. if $W_{1}=0$ but $W_{2} \neq 0$ then the origin is a second order fine focus whose stability is determined by $\operatorname{sgn} W_{2}$;

c. if $W_{1}=W_{2}=0$ but $W_{3} \neq 0$ then the origin is a third order fine focus whose stability is determined by $\operatorname{sgn} W_{3}$;

d. if $W_{1}=W_{2}=W_{3}=0$ but $W_{4} \neq 0$ then the origin is a fourth order fine focus whose stability is determined by $\operatorname{sgn} W_{4}$; 
e. if $W_{1}=W_{2}=W_{3}=W_{4}=0$ then the origin is a center;

$f$. the origin is a center iff $c_{02}+\lambda \eta=2 c_{20}-\lambda c_{11}+2 \lambda \eta=0$ or $c_{20}=c_{11}=$ $c_{02}=0$.

Proof. Addressing point (f) first, by a time rescaling we replace (4.1) with the polynomial system

$$
\begin{aligned}
\dot{u} & =v\left(1+\eta u^{2}\right) \\
\dot{v} & =(-u-u w)\left(1+\eta u^{2}\right) \\
\dot{w} & =-\lambda w\left(1+\eta u^{2}\right)+c_{20} u^{2}+c_{11} u v+c_{02} v^{2}
\end{aligned}
$$

which has the same oriented trajectories as (4.1). The first four nonzero focus quantities were computed by means of the method described in Section 2, first complexifying (4.2) and then computing as described earlier. The first two nonzero focus quantities, for example, are

$$
\begin{aligned}
& g_{220}=\frac{2 c_{20}-2 c_{02}-\lambda c_{11}}{4+\lambda^{2}} \\
& g_{330}=\frac{\left(c_{20}+c_{02}\right)\left[c_{11} \lambda\left(12+\lambda^{2}\right)-2 c_{02}\left(-4+\lambda^{2}\right)-2 c_{20}\left(12+\lambda^{2}\right)-4 \lambda\left(4+\lambda^{2}\right) \eta\right]}{4 \lambda\left(4+\lambda^{2}\right)^{2}}
\end{aligned}
$$

Let $\widetilde{g}_{k k 0}$ denote the numerator of $g_{k k 0}$ and $\mathcal{B}_{5}=\left\langle\widetilde{g}_{220}, \widetilde{g}_{330}, \widetilde{g}_{440}, \widetilde{g}_{550}\right\rangle$. Using the special-purpose computer algebra system Singular to decompose the radical of $\mathcal{B}_{5}$ into a unique intersection of prime ideals, we obtain the irreducible decomposition of $\mathbf{V}\left(\mathcal{B}_{5}\right)$ as the union of five components $\mathbf{V}\left(J_{j}\right)$, where the ideals $J_{j}$ are:

$$
\begin{aligned}
& J_{1}=\left\langle c_{02}+\lambda \eta, 2 c_{20}-\lambda c_{11}-2 c_{02}\right\rangle \\
& J_{2}=\left\langle\lambda^{2}+4,2 c_{20}-\lambda c_{11}-2 c_{02}\right\rangle \\
& J_{3}=\left\langle 9 \lambda^{2}+4, \ldots, 2 c_{20}-\lambda c_{11}-2 c_{02}\right\rangle \\
& J_{4}=\left\langle\lambda^{2}+1, \ldots, 2 c_{20}-\lambda c_{11}-2 c_{02}\right\rangle \\
& J_{5}=\left\langle c_{11}, c_{02}, 2 c_{20}-\lambda c_{11}-2 c_{02}\right\rangle .
\end{aligned}
$$

Since system (1.1) is real, we get the necessary condition

$$
c_{02}+\lambda \eta=2 c_{20}-\lambda c_{11}+2 \lambda \eta=0 \quad \text { or } \quad c_{20}=c_{11}=c_{02}=0
$$

for the origin to be a center for $\mathfrak{X} \mid W^{c}$.

When there are no nonlinearities in $\dot{w}$ there is a linear center on the unique center manifold the $(u, v)$-plane. When the conditions $c_{02}=2 c_{20}-\lambda c_{11}=$ $-2 \lambda \eta$ hold we look for an invariant algebraic surface $F(u, v, w)=0$ tangent to the $(u, v)$-plane to play the role of the center manifold, in imitation of the case of polynomial feedback. That is, we look for polynomials $F(u, v, w)$ 
and $K(u, v, w)$ that satisfy (2.6) where $\mathfrak{X}$ is the polynomial vector field that corresponds to (4.2) and $K$ is now a polynomial of degree up to three. Equation (2.6) is a polynomial equation in many variables that we were able to solve with Singular, but only over the finite field of characteristic 32003 and with the specific choices $\lambda=1, \eta=4$, and $c_{11}=3$. This solution gave us enough of an indication as to what might be true in the general case that we were able to correctly predict the form of $F$ and $K$ and obtain the general algebraic invariant surface $c_{11} u^{2}-2 \eta u^{2}-2 \eta v^{2}-2 w-2 \eta u^{2} w=0$. The flow of (4.2) restricted to this center manifold is

$$
\dot{u}=\left(1+\eta u^{2}\right) v, \quad \dot{v}=-u-\frac{c_{11}}{2} u^{3}+\eta u v^{2} .
$$

This system is not Hamiltonian but is invariant under the involution $u \rightarrow$ $-u, v \rightarrow v, t \rightarrow-t$, hence is time-reversible, so the origin is a center. This proves part (f).

To prove the other parts of the theorem, instead of computing the Lyapunov quantities for $\mathfrak{X} \mid W^{c}$ as we did in the proof of Theorem 3.2 we compute the Poincaré-Lyapunov quantities. These computations are much more efficient, although the former approach made it immediately apparent that $\eta_{2 k} \equiv 0$ for $1 \leq k \leq 4$, thereby simplifying the proof of Theorem 3.5 somewhat. The computations are even more efficient if we first complexify $\mathfrak{X} \mid W^{c}$, for then the computation is precisely that of the focus quantities of the twodimensional system, which when expressed in the original real parameters give the Poincaré-Lyapunov quantities (compare (2.5), and recall ([13], Remark 3.4.7) that when a system on $\mathbb{C}^{2}$ is the complexification of a real system the focus quantities are real).

In actually implementing these ideas we begin with the observation that by the same reasoning as given in the proof of Lemma 3.1 for the polynomial Moon-Rand system, for the rational Moon-Rand system it is still true that $p_{j k}=0$ when $j+k$ is odd. We compute the first four focus quantities for $\mathfrak{X} \mid W^{c}$,

$$
\begin{aligned}
& \dot{u}=v \\
& \dot{v}=-u-u \sum_{\substack{2 \leq j+k \leq 8 \\
j+k \text { even }}} p_{j k} u^{j} v^{k}
\end{aligned}
$$

but without computing the coefficients $p_{j k}$ in the expansion of the center manifold in terms of the original parameters just yet. 
The first focus quantity $g_{22}$ is the Poincaré-Lyapunov quantity

$$
L_{4}=-\frac{1}{4} p_{11}
$$

When it is zero the second focus quantity $g_{44}$ is the Poincaré-Lyapunov quantity

$$
L_{6}=-\frac{1}{8}\left(p_{31}+p_{13}\right)
$$

When the first two focus quantities are zero, implemented by setting $p_{13}=$ $-p_{31}$, the third is the Poincaré-Lyapunov quantity

$$
L_{8}=-\frac{1}{64}\left(5 p_{51}+3 p_{33}+5 p_{15}-p_{31}\left(5 p_{20}+p_{02}\right)\right) .
$$

When the first three focus quantities are zero, implemented by setting $p_{33}=$ $\left(p_{31}\left(5 p_{20}+p_{02}\right)-5 p_{51}-5 p_{15}\right) / 3$, the fourth is the Poincaré-Lyapunov quantity

$$
\begin{aligned}
L_{10}=-\frac{1}{128}\left(7 p_{17}+5 p_{15} p_{20}\right. & +p_{04} p_{31}+7 p_{20}^{2} p_{31}-p_{22} p_{31}+3 p_{35}-7 p_{31} p_{40}-7 p_{20} p_{51} \\
& \left.-p_{02}\left(-3 p_{15}-p_{20} p_{31}+p_{51}\right)+3 p_{53}+7 p_{71}\right) .
\end{aligned}
$$

Using an initial segment of the geometric series to express the nonlinearities in the right hand side of the $\dot{w}$ equation in powers of $u$ and $w$, we now express the $p_{j k}$ in terms of $\lambda, \eta, c_{20}, c_{11}$, and $c_{02}$. By straightforward computation we obtain

$$
L_{4}=\frac{2 c_{20}-2 c_{02}-\lambda c_{11}}{4\left(4+\lambda^{2}\right)}
$$

which has the sign of $W_{1}$. Since $\eta_{2 k-1} \sim L_{2 k}$, this establishes part (a).

When $L_{4}=0$, implemented by the substitution $c_{02}=c_{20}-\lambda c_{11} / 2$, then

$$
L_{6}=-\frac{\left(4 c_{20}-\lambda c_{11}\right)\left(2 c_{20}-\lambda c_{11}+2 \lambda \eta\right)}{16 \lambda\left(4+\lambda^{2}\right)} \quad\left(\text { when } L_{4}=0\right)
$$

which has the sign of $W_{2}$, so that when $W_{2} \neq 0$ the origin is a second order fine focus whose asymptotic stability is indicated by the sign of $W_{2}$, establishing part (b). If $L_{6}$ is zero because the factor $2 c_{20}-\lambda c_{11}+2 \lambda \eta$ vanishes, then $W_{j}=0$ for $1 \leq j \leq 4$ and $L_{4}=0$ implies that $c_{02}=-\lambda \eta$ so that by part (f) the origin is a center.

When $L_{4}=L_{6}=0$ because $4 c_{20}-\lambda c_{11}=0$ then

$$
L_{8}=-\frac{\lambda c_{11}\left(c_{11}-4 \eta\right)\left(\left(\lambda^{2}+10\right) c_{11}+64 \eta+4 \lambda^{2} \eta\right)}{512\left(4+\lambda^{2}\right)\left(16+\lambda^{2}\right)} \quad\left(\text { when } L_{4}=L_{6}=0\right)
$$


which has the sign of $W_{3}$, and part (c) follows. If $L_{8}$ is zero because $c_{11}\left(c_{11}-\right.$ $4 \eta)=0$ then $W_{j}=0$ for $1 \leq j \leq 4$ and it is easy to check that by part (f) the origin is a center.

When $L_{4}=L_{6}=L_{8}=0$ because $\left(\lambda^{2}+10\right) c_{11}+64 \eta+4 \lambda^{2} \eta=0$ then

$$
L_{10}=\frac{5 \lambda \eta^{4}\left(13+\lambda^{2}\right)\left(16+\lambda^{2}\right)}{8\left(10+\lambda^{2}\right)^{4}} \quad\left(\text { when } L_{4}=L_{6}=L_{8}=0\right)
$$

which has the sign of $W_{4}$, and part (d) follows. $L_{8}=0$ if and only if $\eta=0$, in which case $c_{20}=c_{11}=c_{02}=0$ and the origin is a center. Together with the remarks just above concerning center cases part (e) follows.

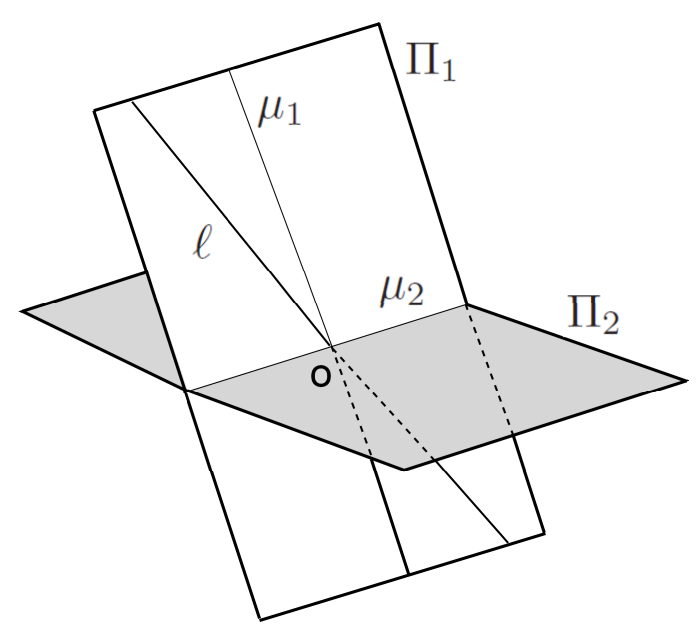

FiguRe 2. Decomposition of the hyperplane $W_{1}=0$ (or $\left.L_{4}=0\right)$ in the $\left(c_{20}, c_{11}, c_{02}, \eta\right)$-parameter space according to stability of the origin.

Figure 2 shows, for any fixed value of $\lambda$, the decomposition of the 3dimensional hyperplane $W_{1}=0$ (or $L_{4}=0$ ) in the 4-dimensional $\left(c_{20}, c_{11}, c_{02}, \eta\right.$ )parameter space according to the stability of the origin of the induced system on any center manifold. $\Pi_{1}$ is the 2 -dimensional plane in $\mathbb{R}^{4}$ defined by

$$
\begin{aligned}
2 c_{20}-\lambda c_{11}-2 c_{02} & =0 & & \left(L_{4}=0\right) \\
4 c_{20}-\lambda c_{11} & =0 & & \left(L_{6}=0 \text { because the first factor is zero }\right)
\end{aligned}
$$


$\Pi_{2}$ is the 2 -dimensional plane in $\mathbb{R}^{4}$ defined by

$$
\begin{aligned}
& 2 c_{20}-\lambda c_{11}-2 c_{02}=0 \quad\left(L_{4}=0\right) \\
& 2 c_{20}-\lambda c_{11}+2 \lambda \eta=0 \quad\left(L_{6}=0 \text { because the second factor is zero }\right)
\end{aligned}
$$

It is one of the two irreducible components of the center variety in $\mathbb{R}^{4}$ for family (4.1). The line $\ell$ is the line in $\mathbb{R}^{4}$ defined by

$$
\begin{aligned}
2 c_{20}-\lambda c_{11}-2 c_{02} & =0 & & \left(L_{4}=0\right) \\
4 c_{20}-\lambda c_{11} & =0 & & \left(L_{6}=0 \text { because the first factor is zero }\right) \\
\left(\lambda^{2}+10\right) c_{11} & =-4\left(16+\lambda^{2}\right) & & \left(L_{8}=0 \text { because the final factor is zero }\right)
\end{aligned}
$$

which can be expressed parametrically by

$$
c_{20}=-\frac{16+\lambda^{2}}{10+\lambda^{2}} \lambda \eta, \quad c_{11}=-4 \frac{16+\lambda^{2}}{10+\lambda^{2}} \eta, \quad c_{02}=\frac{16+\lambda^{2}}{10+\lambda^{2}} \lambda \eta, \quad \eta \in \mathbb{R} .
$$

The line $\mu_{1}$ is the line in $\mathbb{R}^{4}$ defined by

$$
\begin{aligned}
& 2 c_{20}-\lambda c_{11}-2 c_{02}=0 \quad\left(L_{4}=0\right) \\
& 4 c_{20}-\lambda c_{11}-=0 \quad\left(L_{6}=0 \text { because the first factor is zero }\right) \\
& c_{11}=0 \quad\left(L_{8}=0 \text { because } c_{11}=0\right)
\end{aligned}
$$

which can be expressed parametrically by

$$
c_{20}=0, \quad c_{11}=0, \quad c_{02}=0, \quad \eta \in \mathbb{R}
$$

hence is in fact the $\eta$-axis. It is the other of the two irreducible components of the center variety in $\mathbb{R}^{4}$ for family (4.1).

The line $\mu_{2}=\Pi_{1} \cap \Pi_{2}$ (which corresponds to $L_{8}=0$ because $c_{11}=4 \eta$ ) can be expressed parametrically by

$$
c_{20}=-\lambda \eta, \quad c_{11}=4 \eta, \quad c_{02}=\lambda \eta, \quad \eta \in \mathbb{R} .
$$

The point $O$ is the origin of $\mathbb{R}^{4}$.

Outside the 3 -space pictured the origin is a first order fine focus.

Inside the 3 -space pictured but off $\Pi_{1} \cup \Pi_{2}$ (which are always in general position) the origin is a second order fine focus.

In $\Pi_{1}$ but off $\ell \cup \mu_{1} \cup \mu_{2}$ (which are always in general position) the origin is a third order fine focus.

In $\ell$ but not at $O$ the origin is a fourth order fine focus.

To repeat, the center variety is $\Pi_{2} \cup \mu_{1}$. 
Theorem 4.2. Restrict attention to the flow of family (4.1) on a center manifold at the origin.

a. A first order fine focus at the origin has cyclicity zero: no limit cycles bifurcate from it under small perturbation within (4.1).

b. For $2 \leq k \leq 4$, a fine focus of order $k$ at the origin has cyclicity $k-1$ : up to to $k-1$ limit cycles can be made to bifurcate under small perturbation within the family (4.1).

c. Within the family (4.1):

i. A center for which $4 c_{20}-\lambda c_{11} \neq 0$ can be made to bifurcate one limit cycle. (This is $\Pi_{2} \backslash \Pi_{1}$ in Figure 2.)

ii. A center for which $c_{20}=\lambda \eta, c_{11}=4 \eta$, and $c_{02}=-\lambda \eta$ but $\eta \neq 0$ can be made to bifurcate two limit cycles. (This is $\mu_{2} \backslash O$ in Figure 2.)

iii. A center for which $c_{20}=c_{11}=c_{02}=0$ but $\eta \neq 0$ can be made to bifurcate two limit cycles. (This is $\mu_{1} \backslash O$ in Figure 2.)

iv. A center for which $c_{20}=c_{11}=c_{02}=\eta=0$ (the point $O$ in Figure 2) can be made to bifurcate three limit cycles.

Proof. A computation of the Lyapunov quantities for the rational MoonRand system as was done for the polynomial Moon-Rand system shows that it is still true that $\eta_{2}, \eta_{4}$, and $\eta_{8}$ are identically zero, and that $V_{2 k} \sim \eta_{2 k-1}$ for $2 \leq k \leq 5$. Thus the proof follows the same line of reasoning as the proof of Theorem 3.5, the analogous result in the polynomial feedback case, taking the same care in the adjustment of the Lyapunov quantities so as to avoid producing a center.

Remark 4.3. The possibility of creating several limit cycles from the origin means that there exist parameter choices for which, if the parameter values are physically meaningful, the physical system has two asymptotic limits. By bifurcating two limit cycles from a center or a nearby asymptotically stable third order fine focus the polynomial Moon-Rand system can be made to have a coexisting stable equilibrium and stable limit cycle. (Existence of a single stable cycle (and unstable equilibrium) was already observed in the original paper [12] of Moon and Rand by a numerical approximation of the flow.) Although three limit cycles can be made to bifurcate in the rational feedback case, by the mechanism of the proof of the theorem only one can be asymptotically stable, since a fourth order fine focus must be unstable $\left(L_{10}>0\right)$. After the bifurcation the omega limit sets are a stable equilibrium and a stable periodic oscillation. 


\section{The Generalized Polynomial Moon-Rand System}

Consider the following generalization of the polynomial Moon-Rand system (1.1),

$$
\begin{aligned}
\dot{u} & =v \\
\dot{v} & =-u-u w \\
\dot{w} & =-\lambda w+f(u, v), \quad f(u, v)=\sum_{j+k=n} c_{j k} u^{j} v^{k}
\end{aligned}
$$

where $\lambda$ is a nonzero real number. In this section we derive sufficient conditions for the origin to be a center for the flow restricted to a local center manifold, and sufficient conditions for the origin to be asymptotically stable. We start with the center conditions.

5.1. Center conditions. We begin with a simple but important special case.

Proposition 5.1. For the system

$$
\begin{aligned}
\dot{u} & =v \\
\dot{v} & =-u-u w \\
\dot{w} & =-\lambda w+c u^{n}+\frac{n}{\lambda} c u^{n-1} v,
\end{aligned}
$$

the local center manifold $W_{l o c}^{c}$ is unique and is algebraic (in fact is given by the equation $\left.w=(c / \lambda) u^{n}\right)$, and the origin is a center for $\mathfrak{X} \mid W_{\text {loc }}^{c}$.

Proof. The surface $\mathcal{F}(u, v, w)=\lambda w-c u^{n}=0$ is invariant, since $\mathfrak{X} \mathcal{F}=-\lambda \mathcal{F}$ (i.e., the cofactor is $-\lambda$ ). Since it is tangent to the center eigenspace, it is a local center manifold. On the local center manifold the system reduces to

$$
\begin{aligned}
& \dot{u}=v \\
& \dot{v}=-u-\frac{c}{\lambda} u^{n+1},
\end{aligned}
$$

which is Hamiltonian with Hamiltonian function

$$
\mathcal{H}(u, v)=\frac{1}{2}\left(u^{2}+v^{2}\right)+\frac{c}{\lambda(n+2)} u^{n+2} .
$$

Uniqueness of the center manifold is true because it contains a center ([4], $[14])$.

In the following theorem we characterize the existence of a center on the local center manifold when $f(u, v)$ is homogeneous cubic in the special case that $\lambda=1$ (a restriction dictated by computational considerations). 
Theorem 5.2. System (5.1) with $\lambda=1$ and

$$
f(u, v)=c_{30} u^{3}+c_{21} u^{2} v+c_{12} u v^{2}+c_{03} v^{3}
$$

admits a center on the local center manifold at the origin if and only if $c_{12}=c_{03}=3 c_{30}-c_{21}=0$. When it has a center at the origin the local center manifold is unique and algebraic, and can be written $w=\varphi(u, v)=c_{30} u^{3}$.

Proof. The first four nonzero focus quantities are $g_{j j 0}$ for $j=3,6,9,12$. For example $g_{330}$ is

$$
\begin{aligned}
g_{330} & =\left[-294 c_{30}^{2}+35 c_{30} c_{21}+21 c_{21}^{2}-102 c_{30} c_{12}+35 c_{21} c_{12}-4 c_{12}^{2}-45 c_{30} c_{03}\right. \\
& \left.+88 c_{21} c_{03}+55 c_{12} c_{03}+51 c_{03}^{2}\right] / 800 .
\end{aligned}
$$

Because of their size we do not present the others, but they can be easily computed using the method of Section 2.

A decomposition over a field of characteristic zero of the radical of the ideal $\mathcal{B}_{12}=\left\langle g_{j j 0}: j=3,6,9,12\right\rangle$ into an intersection of prime ideals proved to be computationally infeasible, even using the computer algebra system Singular, which is one of the most efficient tools for this kind of computation. The decomposition is possible over the finite field of characteristic 32003, and is:

$$
\begin{aligned}
& J_{1}=\left\langle c_{03}, c_{12}, c_{30}-10668 c_{21}\right\rangle, \\
& J_{2}=\left\langle c_{21}+3 c_{03}, c_{30}+10668 c_{12}, c_{12}^{2}+9 c_{03}^{2}\right\rangle . \\
& \text { Since } 10668 \equiv 1 / 3 \bmod 32003 \text { this is } \\
& \tilde{J}_{1}=\left\langle c_{03}, c_{12}, c_{30}-(1 / 3) c_{21}\right\rangle, \\
& \tilde{J}_{2}=\left\langle c_{21}+3 c_{03}, c_{30}+(1 / 3) c_{12}, c_{12}^{2}+9 c_{03}^{2}\right\rangle .
\end{aligned}
$$

Although conditions obtained using modular arithmetic do not necessarily provide accurate necessary conditions, this computation suggests that in analogy with the quadratic case a center is possible only if $c_{03}=c_{12}=0$.

With this hint, we can verify the necessity of $c_{03}=0$ as follows. If $c_{03} \neq 0$ then a linear change of coordinates of the form $u=U, v=V, w=\gamma W$ exists that preserves the form of (5.1) but with $c_{03}$ replaced by 1 or -1 . A Gröbner basis of the ideal $\mathcal{B}_{12}$ in the special case that $c_{03}= \pm 1$ is

$$
\left\{9+c_{12}^{2}, 3+c_{21}, 3 c_{30}+c_{12}\right\} .
$$

Since we are concerned with real parameter values $\mathbf{V}\left(\mathcal{B}_{12}\right)=\varnothing$, so there is no center on the center manifold. In a similar fashion we show that $c_{12}=0$ 
is necessary for a center. A Gröbner basis of $\mathcal{B}_{12}$ when $c_{12}=c_{03}=0$ is

$$
\left\{\left(3 c_{30}-c_{21}\right) c_{21}^{3},\left(3 c_{30}-c_{21}\right)\left(14 c_{30}+3 c_{21}\right)\right\},
$$

yielding the conditions of the theorem.

Sufficiency follows directly from Proposition 5.1 with $\lambda=1$ and $n=3$, as do the remaining assertions of the theorem.

Conjecture 5.3. System (5.1) with $f$ of the form

$$
f(u, v)=c_{n 0} u^{n}+c_{n-1,1} u^{n-1} v+\cdots+c_{0 n} v^{n} .
$$

admits a center on the local center manifold if and only if $f(u, v)=c u^{n}+$ $\frac{c}{\lambda} n u^{n-1} v$.

Additional evidence for Conjecture 5.3 is given by the following proposition.

Proposition 5.4. System (5.1) with $f(u, v)=\sum_{j+k=n} c_{j k} u^{j} v^{k}$ has an algebraic local center manifold $W_{\text {loc }}^{c}$ expressible in the form $w=\varphi(u, v)=$ $\sum_{j+k=2}^{N} d_{j k} u^{j} v^{k}$ if and only if $f(u, v)=c u^{n}+\frac{c}{\lambda} n u^{n-1} v$. In such a case $\varphi(u, v)=\frac{c}{\lambda} u^{n}$ and the singularity at the origin of $\mathfrak{X} \mid W_{\text {loc }}^{c}$ is a center.

The following lemma will be needed in the proof and for results in the next subsection on aymptotic stability of system (5.1).

Lemma 5.5. Let $M_{n}$ denote the $(n+1) \times(n+1)$ tridiagonal matrix $M_{n}=$ $\left[a_{j k}\right]$ such that $a_{j, j+1}=j, a_{j, j}=-\lambda$, and $a_{j+1, j}=j-(n+1)$ for $1 \leq j \leq n$, $a_{n+1, n+1}=-\lambda$, and $a_{j, k}=0$ for all other pairs $(j, k) . \quad\left(M_{2}\right.$ and $M_{3}$ are displayed in the proof of the proposition.) Then $M_{n}$ is invertible for all nonzero real $\lambda$.

Proof of the lemma. The determinant of $M_{n}$ is a polynomial of degree $n+1$ in $\lambda$.

We note first that if $\lambda_{0}$ is a root of $\operatorname{det} M_{n}$ then so is $-\lambda_{0}$. For let $M_{n}^{-}$ denote the matrix obtained from $M_{n}$ by negating each occurrence of $\lambda$. Then $M_{n}$ is obtained from $M_{n}^{-}$by the following sequence of row operations, each of which either negates the determinant or leaves it unchanged: negate every row, interchange the first and last rows, the second and next to last rows, and so on, and interchange pairs of columns similarly. This implies that $\operatorname{det} M_{n}$ is a polynomial in $\lambda^{2}$ (if $n$ is odd) or is $\lambda$ times such a polynomial (if $n$ is even). 
Secondly, we note that every coefficient in $\operatorname{det} M_{n}$ has the same sign. This is a consequence of the following compound statement that is easily proved by induction, expanding the relevant determinants along the first row:

Suppose $A$ is an $(n+1) \times(n+1)$ tridiagonal matrix such that every element on the superdiagonal is nonnegative, every element on the main diagonal is $-\lambda$, and every element on the subdiagonal is nonpositive; suppose $B$ is an $(n+1) \times(n+1)$ tridiagonal matrix with the same properties, except that the $(1,1)$ entry of $B$ is a nonpositive number. Then the sign of every nonzero coefficient in either of $\operatorname{det} A$ and $\operatorname{det} B$, regarded as polynomials in $\lambda$, is positive if $n$ is odd and is negative if $n$ is even.

The two facts together imply that $\operatorname{det} M_{n}$ is a polynomial in $\lambda^{2}$ (if $n$ is odd) or is $\lambda$ times such a polynomial (if $n$ is even), every nonzero coefficient of which has the same sign. By Descartes' Rule of Signs it has no real roots other than possibly zero.

Proof of the proposition. The local center manifold at the origin can always be realized as $w=\varphi(u, v)$ on a neighborhood of the origin in the $(u, v)$ plane. Inserting the expression $\varphi(u, v)=\sum_{j+k=2}^{N} d_{j k} u^{j} v^{k}$ into the defining equation

$$
v \varphi_{u}(u, v)-(u+u \varphi(u, v)) \varphi_{v}(u, v)=-\lambda \varphi(u, v)+f(u, v)
$$

for the local center manifold and collecting terms yields

$$
\begin{aligned}
& \sum_{j+k=2}^{N}\left[(k+1) d_{j-1, k+1}-(j+1) d_{j+1, k-1}-\lambda d_{j k}\right] u^{j} v^{k} \\
& \quad+\left(\sum_{j+k=2}^{N} d_{j k} u^{j} v^{k}\right)\left(\sum_{r+s=2}^{N}(s+1) d_{r-1, s+1} u^{r} v^{s}\right)=-\sum_{j+k=n} c_{j k} u^{j} v^{k}
\end{aligned}
$$

where it is understood that $d_{\alpha, \beta}$ is replaced by zero if $\alpha<0$ or if $\beta<0$.

We first show that $\varphi$ contains no terms of order less than $n$, hence that $N \geq n$. This is automatic if $n=2$. If $n>2$ the quadratic terms in (5.3) read

$$
\left(\begin{array}{ccc}
-\lambda & 1 & 0 \\
-2 & -\lambda & 2 \\
0 & -1 & -\lambda
\end{array}\right)\left(\begin{array}{l}
d_{20} \\
d_{11} \\
d_{02}
\end{array}\right)=\left(\begin{array}{l}
0 \\
0 \\
0
\end{array}\right)
$$

which by Lemma 5.5 implies that $d_{j k}=0$ for $j+k=2$. 
If $n>3$ the cubic terms in (5.3) read

$$
\left(\begin{array}{cccc}
-\lambda & 1 & 0 & 0 \\
-3 & -\lambda & 2 & 0 \\
0 & -2 & -\lambda & 3 \\
0 & 0 & -1 & -\lambda
\end{array}\right)\left(\begin{array}{l}
d_{30} \\
d_{21} \\
d_{12} \\
d_{03}
\end{array}\right)=\left(\begin{array}{l}
0 \\
0 \\
0 \\
0
\end{array}\right)
$$

which by Lemma 5.5 implies that $d_{j k}=0$ for $j+k=3$.

Successively considering terms of order $m, 4 \leq m \leq n-1$, in (5.3), the product on the left hand side of (5.3) does not contribute because by the previous steps each factor begins with terms of order at least $m$. Thus the terms of order $m$ in (5.3) are

$$
M_{n}\left(d_{m 0}, d_{m-1,1}, \ldots, d_{0 m}\right)^{T}=(0, \ldots, 0)^{T}
$$

where $M_{n}$ is the matrix of Lemma 5.5, which implies that the terms of order $m$ are absent from $\varphi$.

Now successively examine terms of order $2 N, 2 N-1$, and so on in (5.3). The terms of order $2 N$ read

$$
\left(\sum_{j+k=N} d_{j k} u^{j} v^{k}\right)\left(\sum_{r+s=N}(s+1) d_{r-1, s+1} u^{r} v^{s}\right)=0 .
$$

Since the left factor in the product is not zero, the other is, implying that

$$
d_{N-1,1}=d_{N-2,2}=\cdots=d_{0, N}=0
$$

and the $N$ th order terms in $\varphi$ reduce to $d_{N 0} u^{N}$.

The terms of order $2 N-1$ in (5.3) read

$$
\begin{aligned}
& \left(\sum_{j+k=N-1} d_{j k} u^{j} v^{k}\right)\left(\sum_{r+s=N}(s+1) d_{r-1, s+1} u^{r} v^{s}\right) \\
& +\left(\sum_{j+k=N} d_{j k} u^{j} v^{k}\right)\left(\sum_{r+s=N-1}(s+1) d_{r-1, s+1} u^{r} v^{s}\right)=0 .
\end{aligned}
$$

But in the previous step it was shown that $d_{j k}=0$ if $j+k=N$ and $j<N$ so (5.4) reduces to

$$
d_{N 0} u^{N} \sum_{r+s=N-1}(s+1) d_{r-1, s+1} u^{r} v^{s}=0 .
$$

Since $d_{N 0} \neq 0$,

$$
d_{N-2,1}=d_{N-3,2}=\cdots=d_{0, N-1}=0
$$

and the $(N-1)$ st order terms in $\varphi$ reduce to $d_{N-1,0} u^{N-1}$. 
For sufficiently small $\gamma \in \mathbb{N}$ a similar argument applies to terms of order $2 N-\gamma$ in (5.3), for which the analogue of (5.4) is a $(\gamma+1)$-fold sum

$$
\begin{aligned}
& \left(\sum_{j+k=N-\gamma} d_{j k} u^{j} v^{k}\right)\left(\sum_{r+s=N}(s+1) d_{r-1, s+1} u^{r} v^{s}\right)+\cdots \\
& +\left(\sum_{j+k=N} d_{j k} u^{j} v^{k}\right)\left(\sum_{r+s=N-\gamma}(s+1) d_{r-1, s+1} u^{r} v^{s}\right)
\end{aligned}
$$

equal to zero, and shows that the terms of order $N-\gamma$ in $\varphi$ reduce to $d_{N-\gamma, 0} u^{N-\gamma}$. The argument is valid for all $\gamma$ for which (i) $2 N-\gamma \geq N+1$, so that the terms of order $2 N-\gamma$ in the right hand side of (5.3) are zero (the first term on the left in (5.3) does not contribute, nor does the term on the right), and (ii) $N-\gamma \geq n$, so that each term in each product in (5.5) appears initially. The binding condition is that $N-\gamma \geq n$, or $\gamma \leq N-n$. For $\gamma=N-n$ we have the conclusion that terms of order $n$ in $\varphi$ reduce to $d_{n 0} u^{n}$.

The argument so far shows that $\varphi$ must have the form

$$
\varphi(u, v)=d_{n 0} u^{n}+d_{n+1,0} u^{n+1}+\cdots+d_{N 0} u^{N} .
$$

The local center manifold condition (5.2) then reads

$\sum_{j+k=n} c_{j k} u^{j} v^{k}-\lambda\left(d_{n 0} u^{n}+\cdots+d_{N 0} u^{N}\right)-v\left(n d_{n 0} u^{n-1}+\cdots+N d_{N 0} u^{N-1}\right)=0$.

The terms of order other than $n$ that contain $v$ must be absent from the rightmost term, hence $d_{j 0}$ is zero unless $j=n$, and (5.6) reduces to

$$
\sum_{j+k=n} c_{j k} u^{j} v^{k}-\lambda d_{n 0} u^{n}-n d_{n 0} u^{n-1} v=0,
$$

which holds if and only if

$$
c_{n 0}=\lambda d_{n 0}, \quad c_{n-1,1}=n d_{n 0}, \quad c_{j k}=0 \text { otherwise. }
$$

In this case by Proposition 5.1 there is a center on the center manifold, which is given by $w=\varphi(u, v)=d_{n 0} u^{n}$. 
5.2. Asymptotic stability. For the results of this subsection we will treat a slightly more general family

$$
\begin{aligned}
\dot{u} & =v \\
\dot{v} & =-u-u w \\
\dot{w} & =-\lambda w+f(u, v), \quad f(u, v)=\sum_{j+k=n} c_{j k} u^{j} v^{k}+\cdots
\end{aligned}
$$

where $f$ is sufficiently smooth, namely, $C^{n+1}$, and $\lambda$ is a nonzero real number. We begin with a lemma concerning any center manifold.

Lemma 5.6. Let $W^{c}$ be any $C^{n+1}$ center manifold for system (5.7) at the origin, represented by

$$
w=h(u, v)=\sum_{j+k=2}^{n} p_{j k} u^{j} v^{k}+\cdots .
$$

Then $p_{j k}=0$ for $j+k \leq n-1$ and for $r \in\{n, n+1\}$

$$
\left[\begin{array}{c}
p_{r, 0} \\
p_{r-1,1} \\
\vdots \\
p_{0, r}
\end{array}\right]=M_{r}^{-1}\left[\begin{array}{c}
-c_{r, 0} \\
-c_{r-1,1} \\
\vdots \\
-c_{0, r}
\end{array}\right]
$$

where $M_{r}$ is the matrix of Lemma 5.5.

Proof. The defining equation for the local center manifold is

$$
\left.v h_{u}(u, v)-u h_{v}(u, v)+u h(u, v)\right) h_{v}(u, v)+\lambda h(u, v)-f(u, v)=0 .
$$

When the coefficients of the terms of degree two are equated to zero the summand $u h h_{v}$ makes no contribution, so we obtain the system of equations

$$
M_{r}\left[\begin{array}{c}
p_{r, 0} \\
p_{r-1,1} \\
\vdots \\
p_{0, r}
\end{array}\right]=\left[\begin{array}{c}
0 \\
0 \\
\vdots \\
0
\end{array}\right]
$$

with $r=2$. Setting the coefficients of terms of degree three equal to zero, the summand $u h h_{v}$ now begins with terms of order six, hence makes no contribution, so we obtain the system of equations (5.8) with $r=3$. The pattern continues through order $n-1$. At order $n$ the system of equations 
obtained is

$$
M_{r}\left[\begin{array}{c}
p_{r, 0} \\
p_{r-1,1} \\
\vdots \\
p_{0, r}
\end{array}\right]=\left[\begin{array}{c}
-c_{r, 0} \\
-c_{r-1,1} \\
\vdots \\
-c_{0, r}
\end{array}\right]
$$

with $r=n$. For order $n+1$ we obtain similarly (5.9) with $r=n+1$.

Theorem 5.7. Let $\mathfrak{X}$ be vector field associated to system (5.7). Let $W^{c}$ be any $C^{n+1}$ center manifold at the origin. Define the discriminant quantity $W$ by:

if $n$ is even:

$$
\begin{array}{r}
W=-\left[\left(\frac{1}{n+1}\right) p_{n-1,1}+\left(\frac{1 \cdot 3}{(n+1)(n-1)}\right) p_{n-3,3}+\left(\frac{1 \cdot 3 \cdot 5}{(n+1)(n-1)(n-3)}\right) p_{n-5,5}\right. \\
\left.+\cdots+\left(\frac{1 \cdot 3 \cdots(n-5)(n-3)}{(n+1)(n-1) \cdots \cdot 5 \cdot 5}\right) p_{3, n-3}+\left(\frac{1 \cdot 3 \cdots(n-3)(n-1)}{(n+1)(n-1) \cdots 5 \cdot 3}\right) p_{1, n-1}\right]
\end{array}
$$

if $n$ is odd:

$$
\begin{aligned}
W=-\left[\left(\frac{1}{n+2}\right) p_{n, 1}\right. & +\left(\frac{1 \cdot 3}{(n+2) n}\right) p_{n-2,3}+\left(\frac{1 \cdot 3 \cdot 5}{(n+2) n(n-2)}\right) p_{n-4,5} \\
& \left.+\cdots+\left(\frac{1 \cdot 3 \cdots(n-4)(n-2)}{(n+2) n \cdots \cdot 7 \cdot 5}\right) p_{3, n-2}+\left(\frac{1 \cdot 3 \cdots(n-2) n}{(n+2) n \cdots 5 \cdot 3}\right) p_{1, n}\right]
\end{aligned}
$$

where the $p_{j k}$ are the lowest or next to lowest order terms in the expansion of $W^{c}$ as given by Lemma 5.6. Then the origin is asymptotically stable for $\mathfrak{X} \mid W^{c}$ if $W<0$ and is unstable if $W>0$.

Proof. In local coordinates $\mathfrak{X} \mid W^{c}$ is expressed as

$$
\dot{u}=v, \quad \dot{v}=-u-\sum_{j+k=n} p_{j k} u^{j+1} v^{k}+\cdots .
$$

The quantity $W$ is the first Lyapunov quantity that can possibly be non-zero. For simplicity of exposition we will terminate all sums at some indeterminate but sufficiently large number $\alpha$. For

$$
V(u, v)=\frac{1}{2}\left(u^{2}+v^{2}\right)+\sum_{r+s=3}^{\alpha} v_{r s} u^{r} v^{s}
$$


we write

$$
\begin{aligned}
\dot{V}(u, v) & =\left[\sum_{r+s=3}^{\alpha} r v_{r s} u^{r-1} v^{s+1}-\sum_{r+s=3}^{\alpha} s v_{r s} u^{r+1} v^{s-1}\right] \\
& +\left[-\sum_{j+k=n}^{\alpha} p_{j k} u^{j+1} v^{k+1}-\left(\sum_{r+s=3}^{\alpha} s v_{r s} u^{r} v^{s-1}\right)\left(\sum_{j+k=n}^{\alpha} p_{j k} u^{j+1} v^{k+1}\right)\right] \\
& =\sum_{m=3}^{\alpha} \mathcal{L}_{(-R)}\left(V_{m}\right)+Z
\end{aligned}
$$

where $V_{m}$ is the degree- $m$ homogeneous part of $V$ and $\mathcal{L}_{(-R)}$ is the linear operator from the $(m+1)$-dimensional vector space $\mathcal{H}_{m}$ of degree- $m$ homogeneous polynomials in $u$ and $v$ into itself defined by $\mathcal{L}_{(-R)}(p)=v p_{u}-u p_{v}$. It is well known that if $m$ is odd then $\mathcal{L}_{(-R)}$ is a linear isomorphism, while if $m$ is even it has a one-dimensional kernel spanned by $\left(u^{2}+v^{2}\right)^{m / 2}$, and that the kernel is a vector space complement to the range of $\mathcal{L}_{(-R)}([6, \S 8.3])$. We choose the ordered basis $\left\{u^{m}, u^{m-1} v, \ldots, u v^{m-1}, v^{m}\right\}$ for $\mathcal{H}_{m}$, with respect to which the matrix representative of $\mathcal{L}_{(-R)}$ is the matrix $M_{m}$ of Lemma 5.5 with $\lambda=0$, which we denote by $M_{m}^{0}$. The coordinate vector of an element $\xi$ of $\mathcal{H}_{m}$ will be denoted $[\xi]$. In particular for even $m$ we write

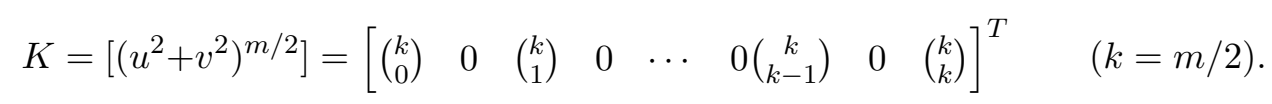

Since $Z$ begins with terms of degree $n+2$, for the homogeneous terms of degree $m \in\{3,4, \ldots, n+1\}$ zeroing the coefficients gives the system of linear equations

$$
M_{m}^{0}\left[\begin{array}{c}
v_{m 0} \\
\vdots \\
v_{0 m}
\end{array}\right]=0 .
$$

If $m$ is odd the unique solution is the zero vector. There is no advantage in not choosing the same solution when $m$ is even. We note for future reference that since $n \geq 2$ it is always the case that $v_{30}=v_{21}=v_{12}=v_{03}=0$. In any event

$$
\dot{V}(u, v)=\mathcal{L}_{(-R)}\left(V_{m}\right)+Z_{m}+\stackrel{(m+1)}{\cdots}
$$

where

$Z_{m}=-\left(p_{m-2,0} u^{m-1} v+p_{m-3,1} u^{m-2} v^{2}+\cdots+p_{1, m-3} u^{2} v^{m-2}+p_{0, m-2} u v^{m-1}\right)$. 
Suppose $m=n+2$ is even. Then there exists $V_{m} \in \mathcal{H}_{m}$ and a constant $L_{m}$ (in fact the Lyapunov constant we seek) such that

$$
\mathcal{L}_{(-R)}\left(V_{m}\right)-L_{m}\left(u^{2}+v^{2}\right)^{m / 2}=-Z_{m}
$$

so that

$$
\dot{V}(u, v)=L_{m}\left(u^{2}+v^{2}\right)^{m / 2}+\stackrel{(m+1)}{\cdots} .
$$

Writing the matrix $M_{m}^{0}$ as columns $\left[\begin{array}{llll}C_{1} & C_{2} & \cdots & C_{m+1}\end{array}\right]$, its column space is $\operatorname{span}\left\{C_{1}, \cdots, C_{m}\right\}$. Since $\operatorname{ker}\left(\mathcal{L}_{(-R)}\right)$ is a vector space complement to Image $\left\{\mathcal{L}_{(-R)}\right\}$ the matrix $\left[\begin{array}{llll}C_{1} & \cdots & C_{m} & K\end{array}\right]$ is invertible. Letting $c_{j}$ denote the vector whose coordinate vector is $C_{j}$ and $r_{1}, \ldots, r_{m}$ constants such that $r_{1} c_{1}+\cdots+r_{m} c_{m}=\mathcal{L}_{(-R)}\left(V_{m}\right)$, by $(5.12)$

$$
\left[\begin{array}{llll}
C_{1} & \cdots & C_{m} & K
\end{array}\right]\left[\begin{array}{c}
r_{1} \\
\vdots \\
r_{m} \\
-L_{m}
\end{array}\right]=\left[-Z_{m}\right] .
$$

Thus $L_{m}$ is the last row of $\left[\begin{array}{lllll}C_{1} & \cdots & C_{m} & K]^{-1} \text { times }\left[Z_{m}\right.\end{array}\right]$.

One can verify by inspection that up to rescaling by a positive constant the last row of $\left[C_{1} \cdots C_{m} K\right]^{-1}$ is

$$
\left[\begin{array}{lllllllllll}
1 & 0 & \frac{1}{m-1} & 0 & \frac{1 \cdot 3}{(m-1)(m-3)} & 0 & \cdots & 0 & \frac{1 \cdot 3 \cdots(m-5)(m-3)}{(m-1)(m-3) \cdots 3} & 0 & \frac{1 \cdot 3 \cdots(m-3)(m-1)}{(m-1)(m-3) \cdots 3 \cdot 1}
\end{array}\right] .
$$

Since by (5.11)

$$
\left[Z_{m}\right]=\left(\begin{array}{llllllll}
0 & -p_{m-2,0} & -p_{m-3,1} & \cdots & p_{2, m-4} & p_{1, m-3} & p_{0, m-2} & 0
\end{array}\right)^{T}
$$

the result follows for the case $n$ even.

Suppose $m=n+2$ is odd. Then there exists $V_{m} \in \mathcal{H}_{m}$ such that $\mathcal{L}_{(-R)}\left(V_{m}\right)=-Z_{m}$. For this choice of $V_{m}$ the lowest order terms in $\dot{V}$ are order $m+1=n+3$; by (5.10) they are

$$
\begin{aligned}
\mathcal{L}_{(-R)}\left(V_{m+1}\right)- & \left(p_{n+1,0} u^{n+2} v+p_{n, 1} u^{n+1} v^{2}+\cdots+p_{1, m-2} u^{2} v^{n-1}+p_{0, n+1} u v^{n+2}\right) \\
- & \left(v_{21} u^{2}+2 v_{12} u v+3 v_{03} v^{2}\right) \times \\
& \left(p_{n, 0} u^{n+1}+p_{n-1,1} u^{n} v+\cdots+p_{1, n-1} u^{2} v^{n-1}+p_{0, n} u v^{n}\right) .
\end{aligned}
$$

But as noted above $v_{j k}=0$ for $j+k=3$ so

$$
\dot{V}=\mathcal{L}_{(-R)}\left(V_{m+1}\right)+Z_{m+1}+\stackrel{(m+1)}{\cdots}
$$


for

$Z_{m+1}=-\left(p_{m-1,0} u^{m} v+p_{m-2,1} u^{m-1} v^{2}+\cdots+p_{1, m-2} u^{2} v^{m-1}+p_{0, m-1} u v^{m}\right)$.

The discussion of the previous case applies but with $m$ replaced by $m+1$.

Remark 5.8. We note the interesting fact that when the lowest order nonlinearities in the $\dot{w}$ equation in system (5.7) are of odd order then they do not play a role in the asymptotic stability of the origin for $\mathfrak{X} \mid W^{c}$.

\section{REFERENCES}

[1] Aulbach, B. A classical approach to the analyticity problem of center manifolds, Z. Angew. Math. Phys. 36 (1985) $1-23$.

[2] Bautin, N. N. Du nombre de cycles limites naissant en cas de variation des coefficients d'un état d'équilibre du type foyer ou centre, Dokl. Akad. Nauk SSSR 24 (1939) 669672 .

[3] Burchard, A., B. Deng, and K. Lu. Smooth conjugacy of center manifolds, Proc. Roy. Soc. Edinburgh Sect. A 120 (1992) 61-77.

[4] Bibikov, Y. N. Local Theory of Nonlinear Analytic Ordinary Differential Equations. Lecture Notes in Mathematics, Vol. 702. New York: Springer-Verlag, 1979.

[5] Carr, J. Applications of Centre Manifold Theory. Applied Mathematical Sciences, Vol. 35. New York: Springer-Verlag, 1981.

[6] Chicone, C. Ordinary Differential Equations with Applications. New York: SpringerVerlag, 1999.

[7] Decker, W., G. Pfister, and H. Schnemann. primdec.lib. A Singular 3-0-4 library for computing the primary decomposition and radical of ideals, 2007.

[8] Edneral, V., A. Mahdi, V. G. Romanovski, and D. S. Shafer. The center problem on a center manifold in $\mathbb{R}^{3}$, Nonlinear Analysis A 75 (2012) 2614-2622.

[9] Greuel, G.-M., G. Pfister, and H. Schnemann. Singular 3-0-4. A computer algebra system for polynomial computations. http://www.singular.uni-kl.de, 2007.

[10] Kuznetsov, Y. A. Elements of applied bifurcation theory, Third edition. New York: Springer-Verlag, 2004.

[11] Lassalle, M. G. Une démonstration du théorème de division pour les fonctions différentiables, Topology 12 (1973) 41-62.

[12] Moon, F. C., and R. H. Rand. Parametric stiffness control of flexible structures, Jet Propulsion Laboratory Publication 85-29, Vol. II, California Institute of Technology (1985)329-342.

[13] Romanovski, V. G., and D. S. Shafer. The Center and Cyclicity Problems: A Computational Algebra Approach. Boston: Birkhäuser, 2009.

[14] J. Sijbrand. Properties of center manifolds, Trans. Amer. Math. Soc. 289 (1985) $431-469$. 
Mathematics Department, North Carolina State University, Raleigh, NC 27695, USA

E-mail address: amahdi@ncsu.edu

Center for Applied Mathematics and Theoretical Physics, University of Maribor, Kerkova 2, Si-2000 Maribor, Slovenia and Faculty of Natural Sciences and Mathematics, University of Maribor, KorošKa Cesta 160, SI-2000 Maribor, Slovenia

E-mail address: valery.romanovsky@uni-mb.si

Mathematics Department, University of North Carolina at Charlotte, Charlotte, North Carolina 28223, USA

E-mail address: dsshafer@uncc.edu 